\title{
Review of Methods for Automatic Segmention of Brain Tumor in MRI Images
}

\author{
Narapareddy Ramarao \\ Department of EEE, \\ B M S Institute of Technologyand Management, \\ Bangalore, India \\ Deepa M. S \\ Department of EEE, \\ BMS Institute of Technology and Management, \\ Bangalore, India
}

\author{
Kavya $\mathrm{P}$ \\ Department of EEE, \\ BMS Institute of Technology and Management, \\ Bangalore, India \\ Sinchana Gowda \\ Department of EEE, \\ BMS Institute of Technology and Management, \\ Bangalore, India
}

\author{
Vaishnovi H \\ Department of EEE, \\ BMS Institute of Technology and Management, \\ Bangalore, India
}

\begin{abstract}
Efficient early detection of Brain tumors enable effective therapy thereby increasing survival rates. Even with growing progress in medical technology, brain tumor detection is an extremely complex task. The segmentation, detection, and extraction of infected tumor area manually from magnetic resonance (MR) images are effective but is a tedious and time taking task performed by radiologiest, which is based on their experience in the field. There are numerous automatic techniques which help in detection of brain tumor with higher degree of accuracy. However, the process of automatic detection and classification varies from technique to technique. Currently there is no particular reliable method widely accepted for tumor detection therefore automatic and reliable methods for tumor detection are of great need and interest. This review paper provides an overview of different methodologies practiced and the best technique adopted for higher accuracy in detecting tumors.
\end{abstract}

Keywords-Pre-processing; Feature extraction;segmentation; Brain tumor detection; MRI image;

\section{INTRODUCTION}

Brain Tumor, the word tumor referred to as neoplasm, means the abnormal expansion of the tissues. Brain tumor can be an abnormal mass of tissue through which cells grow and multiply uncontrollably, unchecked by the mechanisms that control normal cells.Tumors are of two types, Benign and malignant. According to the World health organization, there are different grades( 1 to 4$)$ for these tumors. Tumors of grade 1 and 2 are low-grade tumors while the Grade 3 and 4 means the tumor is of malignant type and is high-graded tumor and this may spread to other parts of the brain and spinal cord and can be fatal. [1].

Nowadays, brain tumor is one of the main reasons for increasing mortality. As the number of patients to treat increase, tools and methods to detect, extract the tumors and also to analyze their behavior exponentially increase. Magnetic Resonance Imaging (MRI) is used to obtain highquality images from all over the body and tissue and to ability of the MRI to detect the smallest details within the body. Through high-resolution imaging MRI, we can infer anatomical information and find out where abnormalities are. This technique has a great ability to detect differences in tissue and structures and is better than CT for the detection of the size of the tumor in the brain.

The brain images obtained using MRI are prone to noise and artifacts such as labels and intensity variations during acquisition. In addition, there are many structures in the brain image such as cerebrospinal fluid, grey matter, and white matter and skull tissues apart from the tumor. Thus, the output MRI Images need to be subjected to a few processes in order to obtain accurate location of the tumor using automatic techniques.

Figure 1 shows the stages in the brain tumor detection and they are[12]:

1. Image Preprocessing

2. Image Segmentation

3. Feature Extraction

4. Feature Selection

5. Classification Algorithms

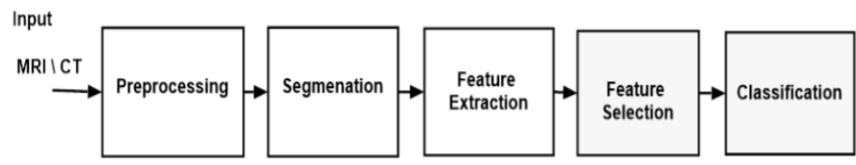

Figure 1- Stages of Brain tumor detection

Preprocessing is the primary step that is applied on MRI image. This step is implemented either to reduce the noise,to reconstruct an imagethen some morphological operations are applied to detect the tumor in the image.Image segmentation is the process of segregating a digital image into segments based on similar characteristics.A label is assigned to every pixel in an image so that pixels with the same label share certain characteristics. In feature extraction, an initial set of raw data is reduced to more manageable groups for processing. 
Feature selection is for filtering irrelevant or redundant features from the dataset.And finally, Classification is to categorize the tumors. All these stages involve different techniques which can be adopted.

\section{PROPOSED METHODS}

The different existing methods are studied to detect the tumor in brain using MRI image. To detect a tumor first thing is to get the MRI scanned image, then preprocessing and enhancement is done. On preprocessed image,segmentation is applied to identify the abnormal portion of the image. Then feature extraction is used to reduce set of features. At last different classifier is applied to detect and classify the tumor.

\section{A. Image Processing and Enhancement}

In pre-processing different techniques are applied such as image enhancement, scale changing, noise removal etc. Each frame of the image is converted from RGB to Gray. Gray scale image enhancement aim is to improve perception of details in images preparing the images suitable for further process. Contrast enhancement process is used to improve the visual details of the image. It has grouped into two i.e. direct and indirect methods.

Spatial resolution and contrast of the image are affected by the noise. Different filters are used to remove noise from the image such as Gaussian filter, median filter and wiener filter. Basic operation of mathematical morphology is that the process of images supported on shapes. There are four mathematical morphology, they are dilation, erosion, opening and closing[2]. An elimination of non-cerebral tissues and unwanted portions of image from the scanned image using a process called Skull striping and is based on threshold operation[3].Histogram equalization process helps in improving the contrast of the image and also which will improve the feature extraction[4]. After image is preprocessed, then the preprocessed image is segmented.

\section{B. Image Segmentation}

The aim of the image segmentation is to divide a medical image into different regions and to extract the area of interest. There are different methods used for segmentation purpose. Region based method used to get the object boundary from the image and this mainly depends on similarity of intensity of pixels in an image and watershed comes under this, it is a transformation defined on a grayscale image. In edge-based segmentation, an edge filter is applied to the image, and then pixels are categorized as edge or non-edge depending on the filter output.

Clustering is important unsupervised learning problem, so it helps in finding a structure in a collection of unlabeled data. The most frequently used clustering algorithms are $\mathrm{K}$ means clustering algorithm and Fuzzy clustering algorithm. $\mathrm{K}$ means algorithm is based on unsupervised technique and human intervention is not required in diagnosing the tumor[5].Fuzzy algorithm used in pattern recognition and fuzzy modeling, is applied for analysis based on space it takes between varieties of input data points[5].Hierarchical segmentation is the region boundaries are maintained at the full image spatial resolution for all segmentations.

Thresholding method is the most often used method. This method is used based on the threshold value to turn a gray scale image into binary image[2][4].Fast Bounding Box Technique (FBB) is an automatic algorithm which is very fast segmentation technique and can locate bounding box which is rectangle axis-parallel around the tumor on MRI image[6] .Graph based method; Region growing method andManual based are the some segmentation methods. Then the feature extraction is done from the segmented image.

\section{Feature Extraction}

The main objective of feature extraction is to minimize the original data set based on the specific characteristics that classify and identify different input patterns. The bestknown feature extractions are local binary pattern (LBP), grey-level co-occurrence matrix (GLCM), canny edge detection, and a bag of words (Bow). A GLCM contains details about the positions of pixels having similar gray level values[1][7]. Classifications of Feature Extraction divide into three main groups i.e. Global Transformation and Series Expansion, ROI Level segmentation, GLCM Texture Features. Global transformation contains Fourier transforms and different wavelet.The discrete wavelet transforms (DWT) being a powerful tool for feature extraction was used[8][9].Discrete wavelet transform is used with extraction of texture and GLCM features in some case.

In some paper SROIs is used with the feature reduction using PCA[10]. GLCM is the most used extraction feature. Once the Feature extraction is done then that image is classified by neural networks and some algorithms.

\section{Classification Technique}

By automatic and semi-automatic techniques identification and separation of the brain tumors can be done. Artificial neural networks are the intelligent based systems. Some of the techniques like Fuzzy C-means, SVM algorithms, Feed Forward Neural Network, KNN algorithm, Self-organizing Map Algorithm etc. are the leading non-intelligent techniques. Better results can be achieved by combining these algorithms. Probabilistic neural network is most often used and it's a feed forward neural network. And it is employed in analysis and pattern recognition problem [1]. Statistical approach is done using ANN[7][10].To classify the detected tumor according to its malignancy level is done by SVM algorithm. SVM algorithm is best approach than KNN \& other algorithm which gives a better accuracy than other. The limitations of neural networks can be overcome by SVM algorithm and the combination of SVM with different neural network gives the best result in classification [3][6].

Different methodologies are studied to detect a brain tumor. By observing we can select a best method which is most accurate and effective to detect a tumor from the MRI image. 


\section{ANALYSIS OF DIFFERENT METHODOLOGIES}

\section{A. Image Preprocessing [11,12]}

There are many techniques used to reduce noise,

\section{Contourlet transform [13]}

This method is used to efficiently approximate images made of smooth regions separated by smooth boundaries. This method is a multi-resolution directional tight frame designed to do the same. This method has a fast implementation based on a laplacian pyramid decomposition followed by directional filterbanks to apply on each bandpass subband.The contourlet transform uses a double filter bank structure so as to get smooth contours of images. In this double filter bank, the Laplacian pyramid (LP) is first used to capture the point discontinuities, after which a directional filter bank (DFB) is used to form those point discontinuities into linear structures.

Advantage

- Used to remove noise in MRI image.

- It removes Gaussian noise present in the MRI image.

- Reduces edge blurring by giving more significance to pixels near the edge.

\section{Disadvantage}

- It is rarely used because of losing required details in image.

- $\quad$ Time consuming[11]

\section{Gaussian filter[13]}

Gaussian filter is a linear filter which is used to blur the images and remove unwanted noise and further detailing.

If two of them are subtracted, it can be used for "unsharp masking" (edge detection). The Gaussian filter alone can be used to blur edges and reduce contrast.The distribution is assumed to have a mean of 0 .

The one advantage a Gaussian filter has over a median filter is that it's faster because multiplying and adding is probably faster than sorting.

Advantage

- Used in directionality, Multi Resolution, localization, critical sampling.

- Its basic functions are Multi-scale and multidimensional.

\section{Disadvantage}

- Highly sensitive to weak boundaries, noise, low contrast between regions, Pseudo Gradients.

- It leads to leakage of boundary or development of false gradient in heterogeneous regions.[11]

\section{Median filter}

The median filter is normally used to reduce noise in an image, similar to the mean filter. However, it does a better job than the mean filter by often preserving useful detail in the image.
Like the mean filter, the median filter also considers each pixel in the image inturn and looks at its nearby neighbours to decide whether or not it is representative of its surroundings. Instead of replacing the pixel value with the mean of neighbouring pixel values, it replaces it with the median of those values thus being more useful than the mean filter.

The median is calculated by first sorting all the pixel values from the surrounding neighbourhood into numerical order and further replacing the pixel being considered with the middle pixel value. If in case the neighbourhood under consideration contains an even number of pixels, the average of the two middle pixel values is used.

Advantage

- Uses nonlinear Noise from the Image that can be extracted using Digital filtering technique.

- It can preserve sharp features in an image. Disadvantage

- $\quad$ It is difficult to analyze.

\section{Anisotropic Diffusion filter}

High-frequency noise is present in magnetic resonance images and needs to be removed by a filtering process. The anisotropic diffusion filter (ADF) is proposed in various filtering applications to adaptively remove the noise, maintaining the image edges. However, when used for the first time, ADF methods still produce unsatisfactory results.

While previous ADF implementations used the gradient of the strongest edges or the standard deviation of a planar region (i.e. without any edges) for filtering, it is proposed a novel approach with improved parameter estimation based on both edge and planar region, overcoming some of ADF important limitations.

Advantage

- Widely used for MRI image enhancement.

- It retains sharp features and edges in the image.

- $\quad$ Reduces contours and noise in Image. Disadvantage

- Not optimal for varying noise.

\section{B. Image Segmentation[11, 12, 13]}

There are many techniques used for segmentation,

\section{K-Means Cluster Algorithm [11]}

In most of the algorithms that are existing K-means Cluster algorithm is one of the extensively used. Kmeans algorithm is one of the simplest and effective ways used in the solution of clustering problems and it is a method that's supported the concept that the gravity centers of the cluster elements represent the cluster. Since this algorithm is mainly based on unsupervised technique.

Hence in this technique human intervention is not required in diagnosing the tumor. It reduces the distance of each element to centre point of the cluster. 
Once the cluster is created it upgrades itself and runs in a loop.

Hence the algorithm will be tested in domains and outcome of the test will be analyzed with standard data sets.

Advantage

- This concept will be useful to treat inputs of the data and analyze data.

- The objects will depend on a variety of data points, location and distance between them.

- Can analyze the regions effectively

Disadvantage

- Not good for noisy, non- uniform and high intensity images inputs.[11]

\section{Fuzzy Clustering algorithm [11,14]}

Fuzzy clustering may be a sort of clustering during which each datum can belong to quite one cluster. It's a sturdy characteristic for ambiguity and may retain far more information than arduous segmentation methods. Fuzzy clustering is successfully used in pattern recognition and fuzzy modeling.

There are various common methods used to categorize classes based on the statistics and the application. Common measures for example distance connectivity and intensity are used. Its usage is in data analysis, pattern recognition and image segments. Most superior method in representing relationship between input pattern pixels and clusters naturally is by using fuzzy clustering. A hard cluster is more flexible than the other methods.

The algorithm is applied to treat annotations of the data and analyze data. The objects are based on the distance between several data points and location. Each cluster is has a center point known as centroid. For overlapping clusters this method will not be successful. Such types of clustering will come under hard clustering.

Advantage

- Can perform better for detecting tumor accurately and approximately.

- Better contrast and good brightness can be achieved.

- This algorithm is applied for analysis based on space it takes between varieties of input data sets.

Disadvantage

- Time taken is moderate

\section{Contour and shape-based methods}

A contour and shape-based method is initially mentioned in proximity to the required boundary. Later this method adjusts the contour along with the destination edge in line with the minimization of predefined specification criteria. An automatic segmentation technique uses past knowledge regards to search out the form of the destination object. This technique starts with a superficial boundary shape which constitutes similar shape of a curve. To spot object boundary from an image it suggested the primary contour and shape-based method. By reducing an energy function the distortion of the contour is restrained. The energy function contains internal and external energy items. The smoothness of the contour is controlled by internal energy. The essential features like axial shape, texture -based information, gradient etc. in the image domain are extracted using contourbased technique.

Advantage

- It will control Cavities, concave-ties, convolution, splitting or merging.

- Tuning is simple and easy.

Disadvantage

- It will add unwanted information like noise in the image.

- It leads to blurring, weak boundaries, edges and reduced contrast.[11]

\section{Region growing method [11]}

Region growing technique is mostly used and implemented technique for image segmentation. This method mainly works on the prior knowledge which is used to extracts the useful information in image region. This relies on intensity details or edges within the image. This may be done by hand selecting a seed point and joining all pixels, in order that all the pixels are connected from initial seed drew on some predetermined criteria.

This idea is to start out with set of seed points of voxels inside the region to be segmented that are manually selected.

The pixels which are approximate have similar gray values and this approach is especially wont to exploit the important factor. This technique is to locate the input image data within the regions which are the set of connected pixels supported the factors which test the properties of the local group of pixels. The pixel are often selected neither by its properties of statistics of its neighborhood nor by its distance from the seed points. This method is free from the seed point. Region growing is also free from noise and all the pixels are interconnected without any discontinuity.

Advantage

- It removes over and under segmentation Disadvantage

- Selection of Seed is difficult.

- Execution time is high and more

5. Graph- based methods[11]

A graph may be a representation of a group of objects, where pairs of the object are connected by the links. It is a mathematical structure and is employed to model pair wise relations between objects. Graph-based method has gained more attention in image segmentation of brain MRI image.

It uses front and backend seeds to locate the brain MRI image objects and with local pixel pair wise similarities enhances the accuracy in graph-based segmentation compared to other methods. It employs many techniques, out of which Graph Cut (GC) is 
most frequently used and along with-it Random walker and Geodesics shortest path methods are some of the other frequently used methods.

Graph cuts are just a minimum cut on a given graph. The goal is to phase the main objects out of an image employing a segmentation method based on graph cuts.

This cut segments images into two regions, because the minimum cut is corresponding to the utmost flow and many graph cut algorithms actually that compute the maximum flow instead of the minimum cut.

Advantage

- It deals with both spatial information and feature features

\section{Disadvantage}

- It fails when multiple objects are used.

\section{Thresholding method}

Threshold-based method is a simple and most effective segmentation technique. This is often the foremost used method and is used based on the threshold value to turn a gray scale image into binary image.

It is a technique for partitioning image directly region based on intensity value or property of these values like intuitive property simplicity of implementation and computational speed. The binary image should contain the information about the position and shape of ROI. By estimating a threshold value for the different regions from the intensity histogram local thresholding can be determined.

So, the edge values of local thresholding are identified by using the local statistical properties like mean intensity value, by the prior knowledge and by calculating partial volumes for each region to determine the brink for the segmentation of each component. And also, the Gaussian distribution was applied to spot the thresholds in normal MRI image. Advantage

- Images with same intensity better performance, high contrast and different gray value among object and background.

- $\quad$ large computational efficiency and accuracy Disadvantage

- Correlation of pixel is not important, because of noise present in the image. [11]

\section{Feature Extraction [11,13,12]}

The best-known feature extractions are

\section{Local Binary Pattern $($ LBP $)$}

It is known for its simplicity and efficiency in texture operation and it labels the pixels of image by thresholding and considers result as binary number. It has discriminative power and computational simplicity.Therefore, LBP texture operator is very popular approach in different applications.

It is considered as combination of the traditional divergent statistics and structural model of the texture analysis. The property of the LBP operator in the real applications which is an important property is that it is robust to the changes caused by monotonic gray-scale like the illumination variations. Other important property is that it is computational simple, that's what makes it to analyze the images in challenging realtime.

Advantage

- Computational simplicity

- Good performance

- High differentiating power

Disadvantage

- Less speed because of long histogram

- Miss out the structure because they do not consider effect of center pixel

- Noise sensitivity is high

2. Grey-level co-occurrence matrix (GLCM) [11]

It is a statistical method that examines the texture which considers spatial relationship of pixels and this is also known as grey -level spatial dependence matrix.

GLCM function characterizes texture of the image that calculates the frequency of occurrence of pairs of pixels with a particular value and in a particular spatial relationship in that image, creates GLCM and then it extracts the statistical measures from that matrix.

Advantage

- Excellent method for image Texture Analysis.

- Textural properties can be calculated to know the details about the image.

- It is a statistical method which is based on the gray level value of pixels

Disadvantage

- Seed selection is more difficult.

- Execution time is high and more.

\section{Canny edge detection}

It is anoperator that uses multi-stage algorithm that detects a wide range of edges in the images. It takes gray scale image as an input and produces image as output which shows the positions of the tracked intensity discontinuities.

It works in multi-stage process. The image is smoothed by Gaussian convolution at first and then the simple 2-D first derivative operator is then applied to the smoothed image that highlights the regions of that image with first spatial derivatives.

Edge gives rise to the ridge in gradient magnitude image. The algorithm is then tracked along the top of those ridges and then it sets all the pixelsto zero that were not on the ridge top and gives a thin line in the output, this process is called as the non-maximal suppression.

The tracking process is exhibited in form of hysteresis which is controlled by the two thresholds: T1 and T2, where $\mathrm{T} 1>\mathrm{T} 2$.

The tracking can only start at a point on the ridge that is higher than that of $\mathrm{T} 1$ and then the tracking continues in both the directions from the point until the height of the ridge falls below threshold T2. This 
hysteresis helps us to understand that the noisy edges are not broken up in to multiple edge fragments.

Advantage

- Immune to noise in environment.

- With the presence of Gaussian filter, it helps to remove noise in image.

- Effectiveness can be adjusted using parameters.

Disadvantage

- It consumes lot of time due to complex computation

- Application on real time systems is difficult.

\section{Bag of words (Bow)}

Bag of words is a very good natural language processing [NLP] technique of text modeling. Wherever this NLP algorithm is applied, it works on numbers. So, we cannot directly feed our text into NLP algorithm. Hence, BOW model is used to preprocess the text by converting it into a bag of words, which will keep an account of total occurrences of the most frequently used words.

Advantage

- Very simple

- Easy to implement

Disadvantage

- It leads to HD vector because of large size vocabulary

- It assumes all the words are independent of each other

\section{Classification techniques [11,12,13,15]}

Some of the techniques like

\section{Fuzzy C-means}

Fuzzy clustering is also called as soft clustering or soft $\mathrm{k}$-means. It is a form of clustering where each data point will belong to more than one cluster.

It involves assigning data points to clusters so that items in the cluster are as similar as possible and items of different clusters are as dissimilar as possible.

So, clusters are identified based on similarity measures. The similarity measures may include distance, connectivity and intensity. The similarity measures may be chosen according to data or application needs.

It is one of the best and most widely used fuzzy clustering algorithm in the fuzzy C-means clustering algorithm.

Advantage

- Gives good result for overlapped data sets

- Efficiency is better than K-means algorithm Disadvantage

- Takes more time because of more iterations involved

- Euclidean distance measures can unequally weight the factors. $[11,14,15]$

\section{SVM algorithms}

SVM algorithm is also called as Support vector machine. It is a supervised learning models that analyze data using classification and regression analysis. It is a popular machine learning tool.

In SVM we plot every data item as a point in ndimensional space, where value of each feature being the value of a particular co-ordinate. Then, the classification is done by finding out hyperplane that will differentiate two classes of data sets. So, SVM is simply the co-ordinates of individual observation.

SVM chooses the extreme points of data sets that are plotted in n-dimensional plot that will help in creating hyperplane. These extreme points are called supportvectors, so the name of the algorithm is SVM algorithm.

Advantage

- It is more effective in higher dimensional spaces

- It is more efficient

- Effective when number of dimension is more than number of samples

\section{Disadvantage}

- Not suitable for large data set

- Do not work well when there is noise in input [11]

\section{Feed Forward Neural Network}

It is also called as Multi-Layered Network of Neurons (MLN). It is called as feed forward network because the information travels only in one direction i.e forward,from input nodes, through the hidden nodes (if any) and to the output nodes. There are no cycles or loops int the network.

It is a type of artificial neural network where connections between the nodes do not form a cycle. It was the first and simplest neural network devised.

Advantage

- Ability to solve more complex representation

- Good for making network deeper in sorting Disadvantage

- Prone to over fitting

- Takes more time $[14,15]$

\section{KNN algorithm}

K-Nearest Neighbor is one of the simplest-algorithm which is used in machine learning. It assumes the similarity between the test data set and the available cases and add the test case into the category that is most similar to the available categories.

This algorithm can be used for both classification and regression analysis but it is most likely to give better output for classification problems. It is a nonparametric algorithm which means it will not make any kind of assumptions for underlying data.

It is also called as lazy learner algorithm because it will not learn for any of the training set available, but it instead it stores the dataset and at the time of classification, it performs action on the dataset. 
This algorithm at the training phase just stores the dataset and when it gets new data, then it classifies the data into the category that is similar to the test data. Advantage

- Simple to implement

- New set of data can be added easily to update the model

- No training period for it

Disadvantage

- Sensitive to noise and missing value

- Does not work well with large set of data

- Does not work well with High dimension data set. $[11,15]$

\section{Self-organizing Map Algorithm}

Self-organizing network is a type of ANN which is trained using unsupervised learning to create a lowdimensional (usually 2D), discrete representation of the input space of training samples, which is called map, so it is the method that does dimensionality reduction.

Self-organizing map differ from other artificial neural network as it applies competitive learning but not error-correction like what different algorithm does.

So, by this SOMs are useful for visualization by creating low-dimensional views of high dimensional data.

It has two layers, i.e input and output layer (reduced map dimension layer). It does not use backpropagation for updating the layers but instead used competitive learning.

\section{Advantage}

- Easy to understand

- Easy to observe similarity in data

- They can be trained in short period of time Disadvantage

- Requires necessary and sufficient data

- Often difficult to obtain perfect mapping [15]

\section{CONCLUSION}

Various pre-processing methodologies are used to reduce the imperfections in the image. Image Re-sampling, gray scale contrast enhancement, noise removal. For noise removal the median filtering technique is used, for image enhancement the edge detection is preferred for the images that have low level of contrast and then post pre processing processes like mathematical operation, skull stripping and histogram equalization are done to get the desired image from input image.

Various segmentation techniques have been explained that include fuzzy clustering means(FCM ), support vector machine(SVM), artificial neural network(ANN), K means clustering, global thresholding, etc are some popular techniques which are used frequently to extract important information from medical imaging modalities. Image thresholding is preferred for the segmentation when the images have high levels of contrast.

Some papers propose BWT and SVM techniques image analysis for MRI-based brain tumor detection and classification which has accuracy of $95 \%$. While some proposed segmentation of MRI brain images using $K$ means clustering algorithm along with morphological filtering for the detection of tumor images as its accuracy is about $95 \%$ and computational time is about 1.2636 (for $\mathrm{k}=3$ ) \&1.1232 sec (for $\mathrm{k}=4$ ). Some proposed a methodology which included extraction of textures features with wavelet transform and SVM with an accuracy of $83 \%$.

The textural features are extracted from gray-level cooccurrence matrix (GLCM) and statistical textural features are extracted from LL and HL sub bands wavelet decomposition and $95 \%$ of accuracy was observed.

A probabilistic neural network (PNN) is widely used in classification and pattern recognition problems because of the advantages like it is faster and more accurate than multilayer perceptron networks, they are comparatively less sensitive to outliers and generate accurate predicted target probability scores. It is considered the most accurate among all the algorithms.

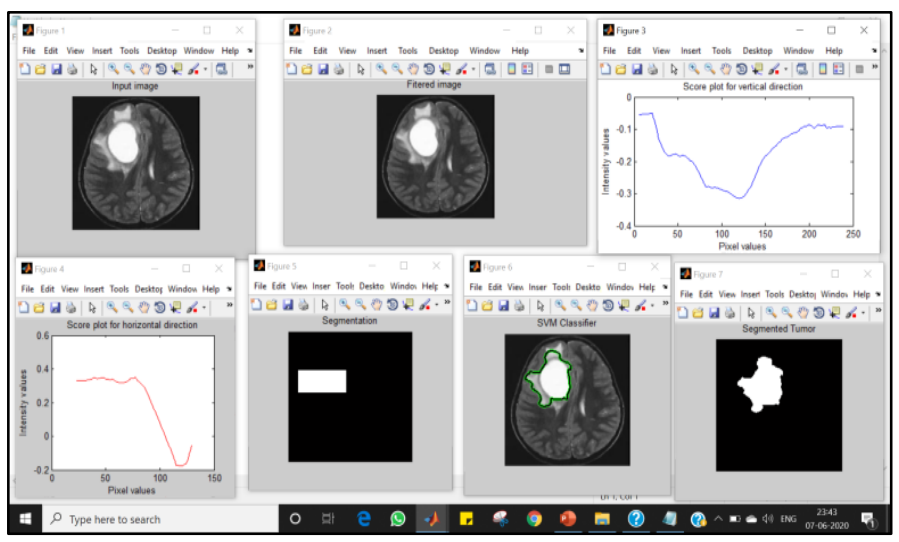

Figure 2 - Results using PNN Algorithm

\section{ACKNOWLEDGEMENT}

The authorsare thankful to the management of BMS Institute of Technology and Management for providing facilities and to publish this work.

\section{REFERENCES}

[1] N. VarunaShreeand T. N. R. Kumar, "Identification and classification of brain tumor MRI images with feature extraction using DWT and probabilistic neural network",Brain Informatics, 8 January 2018, Volume 5, Pages 23-30, DOI: $10.1007 / \mathrm{s} 40708-017-0075-5$

[2] Anupurba Nandi, "Detection of human brain tumor using MRI image segmentation and morphological operators", 2015 IEEE International Conference on Computer Graphics, Vision and Information Security (CGVIS),11 April 2016, DOI: 10.1109/CGVIS.2015.7449892

[3] Niles BhaskarraoBahadure, Arun Kuman Ray, Har Pal Thethi, "Image Analysis for MRI Based Brain Tumor Detection and Feature Extraction Using Biologically Inspired BWT and SVM", International Journal of Biomedical Imaging. 06 Mar 2017, DOI: $10.1155 / 2017 / 9749108$

[4] Abd El Kader Isselmou, Shuai Zhang, Guizhi Xu, "A novel approach for brain tumor detection using MRI Images", Journa of Biomedical Science and Engineering, September 2016 , Volume 9, DOI: 10.4236/jbise.2016.910B006

[5] MahanteshK , Kanyakumari, "BraTS : Brain Tumor Segmentation - Some Contemporary Approaches", International Journal of Innovative Research in Science, May 2016, Volume 5 , ISSN: 2319-8753 
[6] Miss. Rajeshwari G. Tayade, Mr. C.S. Patil, Mr. R. R. Karhe, "Automatic Brain Tumor Detection using SVM and FBB Algorithm",July 2016 International Journal of Scientific Development and Research (IJSDR)Volume 1, Issue 7, ISSN: 2455-2631

[7] Neeraj Sharma, Amit K. Ray, Shiru Sharma, K. K. Shukla, Satyajit Pradhan, and Lalit M. Aggarwal, "Segmentation and classification of medical images using texture-primitive features: Application of BAM-type artificial neural network", Journal of Medical Physics, 2008, Volume 33, Page 119126,DOI: $10.4103 / 0971-6203.42763$

[8] Ahmad M. Sarhan, "Brain Tumor Classification in Magnetic Resonance Images Using Deep Learning and Wavelet Transform", Journal of Biomedical Science and Engineering, June 2020 ,Volume 13, DOI: 10.4236/jbise.2020.136010

[9] Y.V.Sri Varsha , S.PraylaShyry, "A Novel Approach for Identifying the Stages of Brain Tumor", International Journal of Computer Trends and Technology, 2014, Volume 10, DOI:10.14445/22312803/IJCTT-V10P116

[10] Jainy Sachdeva, Vinod Kumar, Indra Gupta, Niranjan Khandelwal, and Chirag Kamal Ahuja, "Segmentation, Feature Extraction, and Multiclass Brain Tumor Classification", Journal of Digital Imaging, 4 May 2013,Volume 26, Page 1141-1150, DOI: $10.1007 / \mathrm{s} 10278-013-9600-0$

[11] G. Sethuram Rao, D. Vydeki, "Brain Tumor Detection Approaches: A Review",2018 International Conference on Smart Systems and Inventive Technology (ICSSIT), 01 July 2019, DOI: 10.1109/ICSSIT.2018.8748692

[12] KalifaShantta and OtmanBasir, "Brain Tumor Detection and Segmentation: A Survey", IRA-International Journal of Technology \& Engineering, Vol.10, Issue 04 Pg.no.55-61. DOI: http://dx.doi.org/10.21013/jte.v10.n4.p1

[13] Ms. Priya Patil , Ms. Seema Pawar , Ms. Sunayna Patil , Prof. Arjun Nichal, "A Review Paper on Brain Tumor Segmentation and Detection", International Journal of Innovative Research in Electrical, Electronics, Instrumentation and Control Engineering, January 2017, DOI: 10.17148/IJIREEICE.2017.5103

[14] Kavitha AngamuthuRajasekaran and ChellamuthuChinnaGounder, "Advanced Brain Tumour Segmentation from MRI Images" ,March 14th 2018,DOI: 10.5772/intechopen.71416

[15] Abd El Kader Isselmou, Guizhi Xu and Shuai Zhang , "Improved Methods for Brain Tumor Detection and Analysis Using MR Brain Images", Biomedical \& Pharmacology Journal, 27 November 2019,DOI : https://dx.doi.org/10.13005/bpj/1793

[16] Hamid Jalab and Ali Majeed Alwaeli , "Magnetic Resonance Imaging Segmentation Techniques of Brain Tumors: A Review "Arch Neurosci. , d 2018 December 12, DOI: 10.5812/ans.84920

[17] Md ShahariarAlam , Md Mahbubur Rahman , Mohammad Amazad Hossain , Md Khairul Islam , KaziMowdud Ahmed , KhandakerTakdir Ahmed, Bikash Chandra Singh , and Md Sipon Miah "Automatic Human Brain Tumor Detection in MRI Image Using Template-Based K Means and Improved Fuzzy C Means Clustering Algorithm", Big Data Cogn. Comput. 13 may 2019, DOI: $10.3390 / \mathrm{bdcc} 3020027$
[18] Sheela.V.K and Dr. S. Suresh Babu "Pre-Processing Technique for Brain Tumor Detection and Segmentation", International Research Journal of Engineering and Technology (IRJET), Volume: 02 ,| June-2015, p-ISSN: 2395-0072

[19] Shubhangi S. Veer (Handore) and Dr. P.M. Patil "An efficient method for Segmentation and Detection of BrainTumor in MRI images ",International Research Journal of Engineering and Technology (IRJET), Volume: 02 , Dec-2015, p-ISSN: 23950072

[20] Faisal Muhammad Shah1, Tonmoy Hossain, Mohsena Ashraf, Fairuz Shadmani Shishir, MD Abdullah Al Nasi andMd. Hasanul Kabir "Brain Tumor Segmentation Techniques on Medical Images - A Review", International Journal of Scientific \& Engineering Research Volume 10, February-2019 ,1514 ISSN 2229-5518

[21] Shilpa B. Sanghavi and Dr. R.J. Shelke "Brain Tumor Classification and Segmentation using PNN \& FCM Clustering", International Journal of Innovative Research in Computer andCommunication Engineering, Vol. 6, April 2018, ISSN (Print) : 2320-9798 